\title{
The Effect of Pulse Vaccination on the Transmission Dynamics of Rotavirus Diarrhea
}

\author{
Amar Nath Chatterjee \\ Department of Mathematics, K. L. S. College, Magadh University, Bodh-Gaya, India \\ Email address: \\ anchaterji@gmail.com \\ To cite this article: \\ Amar Nath Chatterjee. The Effect of Pulse Vaccination on the Transmission Dynamics of Rotavirus Diarrhea. Journal of Chemical, \\ Environmental and Biological Engineering. Vol. 2, No. 1, 2018, pp. 26-31. doi: 10.11648/j.jcebe.20180201.15
}

Received: May 18, 2018; Accepted: June 12, 2018; Published: July 9, 2018

\begin{abstract}
Diarrhea is the third most fatal disease in the developing countries. Approximately 611,000 children die each year due to effect of rotavirus infection. Rotavirus also causes gastroenteritis in adults and it is the main cases of travellers' diarrhea. After initial contact, children are more susceptible to be affected with diarrhoeal illnesses of any kind, but the repeatition of infections with rotavirus tend to be less severe than the original infection. Rotavirus continues to persist, is attributed to the different modes of transmission among the pathogens. To fight against this problem, several rotavirus vaccines have been developed. Federal Drug Administration (FDA) approved drugs within the last two years are currently in use. These vaccines present a degree of protection from rotavirus infection. We formulate a model of the spread of rotavirus diarrhea based on a continuous time ordinary differential equations model. We further expand the model to investigate the effects of pulse vaccination. We use computer simulations to further analyze the effect of vaccination as a controlled method. We find the minimum levels of vaccination necessary in this model to eradicate severe rotavirus disease.
\end{abstract}

Keywords: Diarrhea, Rota Virus, Pulse Vaccination, Impulsive Differential Equation

\section{Introduction}

Rotavirus is a virus for which babies and young children suffer from diarrhea. The common symptoms are vomiting and fever. Due to dehydration and its symptoms including vomiting and watery diarrhea usually last for $3-8$ days. Rotavirus is not the only cause of diarrhea, but it is one of the most common symptoms to diagnose the disease. Worldwide $95 \%$ of children are infected before their 5 years of age and between ages of 4 months to 36 months it reaches its extremity [3]. In developed countries there are fewer deaths associated with rotavirus. But the financial burden of the disease is considerable [2].

The dynamics of rotavirus infections are very complicated. The predominant mode of rotavirus transmission is fecal-oral [5]. The infection spreads through respiratory secretions and person-to-person contacts [6]. The period of incubation of rotavirus disease is near about for 2 days. Contaminated environment is the major cause for the endemic infections but high rates of infections among infants younger than three years old have been identified regardless of sanitary conditions [5].

Rotavirus vaccine is an oral (swallowed) vaccine.
Diarrhoea or vomiting which are caused by other germs will not be prevented by Rotavirus vaccine. But it is very good at preventing diarrhoea and vomiting caused by rotavirus. Most babies who get the vaccine will not get rotavirus diarrhea at all, and almost all of them will be protected from severe rotavirus diarrhea. The development of rotavirus vaccines is used for the control of diarrheal disease in children, and the impact of the introduction of these vaccines at the population-scale is uncertain [3].

A few mathematical studies about Rota virus till today have been addressed as the disease transmission, vaccine and drug options [4-10]. Atkinsa et al. [7] worked on the reduction in RVGE incidence. They observed that if vaccineinduced protection does not wane over three years, severe RVGE in children under five years of age could be eliminated within two years after the introduction of Rota virus vaccination. Skim et al. [8] worked on the role of breast feeding (maternal antibody) on rotavirus control, incorporating the seasonal variation in transmission rate factors. Zelata et al. [9] presented deterministic as well as stochastic models for the transmission of rotavirus in a pediatric hospital ward and draw on published data to compare the efficacy of several possible control measures in 
reducing the number of infections. Roldao et al [10], in their works studied the production of rotavirus virus-like particles (VLP) using the baculovirus expression vector system (BEVS). But control theoretic Mathematical modelling and effect of impulsive vaccination have been received relatively little attention.

This article has been arranged in the following manner.

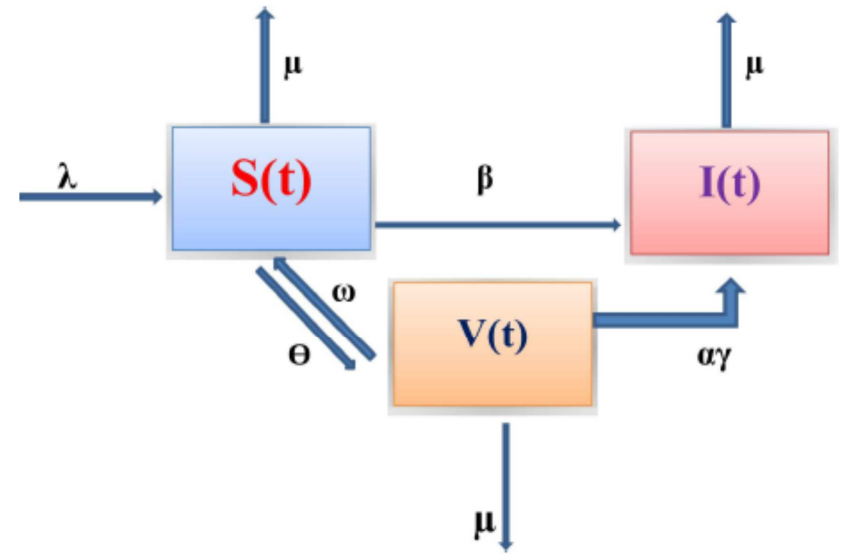

The model without vaccination to examine various dynamics of the system is considered in the section 2. A mathematical model on the basis of perfect drug adherence is analyzed as well as numerical results of this model are discussed in Section 3 and 4 respectively. Implication of the results is discussed in Section 5.

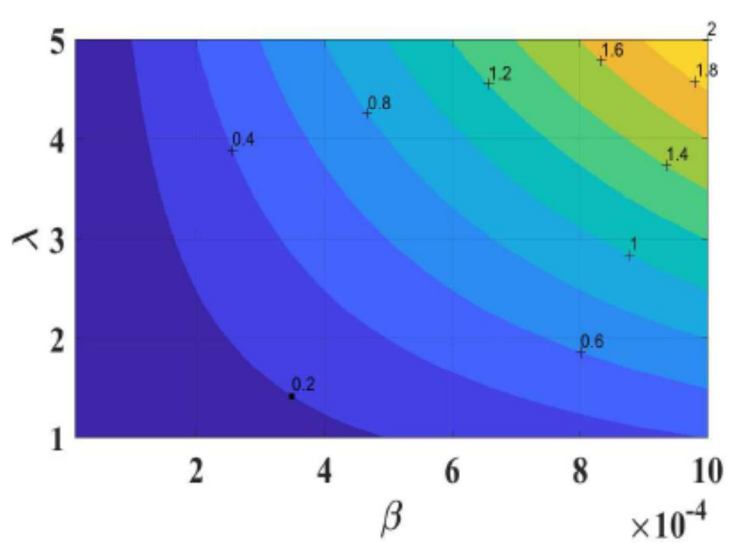

Figure 1. Left Panel: Schematic explanation for the model (1) showing the effect of high drug levels. Right Panel: Contour plot for $R_{0}$ as a function of $\lambda$ and $\beta$.

\section{The System Without Vaccination}

In this section a mathematical model by the effect of rota virus has been propose by considering the three populationsusceptible population (S), Infected population (I) and Vaccinated population (V)respectively. Here the system of ordinary differential equations have been obtained by assuming the population mix homogeneously. Thus, the equation of the model becomes:

$$
\begin{aligned}
& \frac{d S}{d t}=\lambda-\mu S-\beta S I-\theta S+\omega V \\
& \frac{d I}{d t}=\beta S I-\mu I+\alpha v I V \\
& \frac{d V}{d t}=\theta S-\mu V-\omega V-\alpha v I V
\end{aligned}
$$

where the system parameters are defined in the Table 1.

Existence condition and stability analysis of the basic model:

Using the model (1), the disease free equilibrium population values are: $E_{0}=\left(\frac{\lambda(\mu+\omega)}{\mu^{2}+\mu(\omega+\theta)}, 0, \frac{\lambda \theta}{\mu^{2}+\mu(\omega+\theta)}\right)$. The endemic equilibrium values for $E^{*}\left(S^{*}, I^{*}, V^{*}\right)$ are defined below:

$$
S^{*}=\frac{\mu-\alpha v V^{*}}{\beta}, I^{*}=\frac{\theta \mu-(\theta \alpha v+\beta \omega+\beta \mu) V^{*}}{\alpha v \beta V^{*}} \text { and } V^{*} \text { is defined }
$$
from the quadratic equation given below:

$$
\begin{gathered}
(\alpha v \omega-\alpha v \eta) V^{* 2}+\left[\alpha v \lambda+\alpha v \theta \mu+\mu \nu+\alpha^{2} v^{2}(\mu+\theta)\right] V^{*} \\
-\left[\mu^{2} \theta+\mu \alpha v(\mu+\theta)\right]=0,
\end{gathered}
$$

where $\eta=\alpha \theta v+\beta \omega+\beta \omega$.

To study the local stability of the model (1), linearising the system and obtained the following jacobian matrix. $J=$ $\left(\begin{array}{lll}-\mu-\beta I-\theta & \beta S & \omega \\ \beta I & \beta S-\mu S+\alpha \nu V & \alpha \nu I \\ \theta & -\alpha \nu V & -\mu-\omega-\alpha \nu I\end{array}\right)$

For the disease free equilibrium $E_{0}$, the Jacobian becomes

$J\left(E_{0}\right)=\left(\begin{array}{lll}-\mu-\theta & \beta S & \omega \\ \beta I & \beta S-\mu S+\alpha \nu V & \alpha v I \\ \theta & -\alpha \nu V & -\mu-\omega-\alpha v I\end{array}\right)$.

The characteristic equation of the matrix is

$$
(\xi+\mu+\theta)(\xi+\mu+\omega)\left(\xi+\mu-\frac{\beta \lambda}{\mu}\right)=0 .
$$

Eigen values are $\xi_{1}=-(\mu+\theta)<0, \xi_{2}=-(\mu+\omega)<0$, and $\xi_{3}=\frac{\beta \lambda}{\mu}-\mu<0$, if $\frac{\beta \lambda}{\mu^{2}}<1$. All eigen values are negative if the basic reproduction ration $R_{0}=\frac{\beta \lambda}{\mu^{2}}<1$.

Hence it can be concluded that the disease free equilibrium $E_{0}$ stable if the basic reproduction number is less than unity. Other wise the system is unstable.

At the endemic equilibrium $E^{*}$,

$J\left(E^{*}\right)=\left(\begin{array}{lll}-\mu-\beta I^{*}-\theta & \beta S^{*} & \omega \\ \beta I^{*} & \beta S^{*}-\mu S^{*}+\alpha v V^{*} & \alpha \nu I^{*} \\ \theta & -\alpha \nu V^{*} & -\mu-\omega-\alpha \nu I^{*}\end{array}\right)$.

The characteristic equation for $E^{*}$,

$$
\xi^{3}+\eta_{1} \xi^{2}+\eta_{2} \xi+a_{1}\left(a_{2} a_{3}+a_{4}\right)+\eta_{3}=0
$$

where

$$
\begin{gathered}
\eta_{1}=a_{1}+a_{2}+a_{3}>0 \\
\eta_{2}=\left(a_{1} a_{2}+a_{2} a_{3}+a_{1} a_{3}+a_{4}+\beta^{2} S^{*} I^{*}-\omega \theta\right)>0
\end{gathered}
$$




$$
\begin{gathered}
\eta_{3}=a_{1}\left(a_{2} a_{3}+a_{4}\right)+\beta^{2} S^{*} I^{*} a_{3}+\alpha \beta v \theta S^{*} I^{*}+\alpha \beta \nu \omega V^{*} \\
-\omega \theta a_{2}>0,
\end{gathered}
$$

and

$$
\begin{aligned}
& a_{1}=\mu+\theta+\beta I^{*}, \\
& a_{2}=\mu-\beta S^{*}-\alpha v V^{*}, \\
& a_{3}=\mu+\omega+\alpha v I^{*}, \\
& a_{4}=\alpha^{2} v^{2} V^{*} I^{*} .
\end{aligned}
$$

The endemic equilibrium $E^{*}$ is stable if $\eta_{1}>0, \eta_{2}>$ $0, \eta_{1} \eta_{2}-\eta_{3}>0$. Otherwise, the endemic equilibrium $E^{*}$ is unstable.

\section{The System with Vaccination}

Now emphasising the system with pulse vaccination given regular intervals of time say $\tau$, to a proportion of the susceptible human population $p$ the impulsive system, in ordinary differential equation form is illustrated below:

$$
\begin{aligned}
& \frac{d S}{d t}=\lambda-\mu S-\beta S I+\omega V-\theta S \\
& \frac{d I}{d t}=\beta S I-\mu I+\alpha \nu I V \\
& \frac{d V}{d t}=\theta S-\mu V-\omega V-\alpha \nu I V
\end{aligned}
$$

$$
\text { for } t \neq t_{k} \text {. }
$$

The impulsive conditions are given by

$$
\begin{aligned}
& \Delta S=-p S \\
& \Delta V=p S \\
& \text { fort }=t_{k}
\end{aligned}
$$

The susceptible population is considered as $S(t)$, where $f_{1}=\mu+\theta+\beta I$.

$$
\begin{aligned}
\frac{d S}{d t}= & \lambda+\omega V-(\mu+\theta+\beta I) S \\
= & \frac{\lambda\left(\mu^{2}+\mu \omega+\omega \theta\right)}{\mu(\mu+\omega)}-f_{1} S
\end{aligned}
$$

which implies that

$$
\frac{d}{d t}\left(S e^{\int f_{1}(s) d s}\right)=\widehat{\Pi}_{1} e^{\int f_{1}(s) d s}
$$

where $\widehat{\Pi}_{1}=\frac{\lambda\left(\mu^{2}+\mu \omega+\omega \theta\right)}{\mu(\mu+\omega)}$.

Then it follows that, for $t_{k}<t \leq t_{k+1}$,

$$
S(t)=\frac{1}{\left.e^{\int f_{1}(s) d s}\right|_{s=t}}\left[\left.S\left(t_{k}^{+}\right) e^{\int f_{1}(s) d s}\right|_{s=t_{k}}+\int_{t_{k}}^{t} \widehat{\Pi} e^{\int f_{1}(s) d s}\right],
$$

and after setting $t=t_{k+1}$,

$$
S\left(t_{k+1}^{-}\right)=\frac{1}{\left.e^{\int f_{1}(s) d s}\right|_{s=t_{k+1}}}\left[\left.S\left(t_{k}^{+}\right) e^{\int f_{1}(s) d s}\right|_{s=t_{k}^{-}}+\int_{t_{k}}^{t_{k+1}} \widehat{\Pi} e^{\int f_{1}(s) d s}\right]
$$

Let $S\left(t_{0}\right)=\frac{1}{1-p} S(0)$, where $S(0)$ is the initial population of $S(t)$.

Then,

$$
\begin{aligned}
& S\left(t_{1}^{-}\right)=\frac{1}{\left.e^{\int f_{1}(s) d s}\right|_{s=t_{1}}}\left[\left.(1-p) S\left(t_{0}\right) e^{\int f_{1}(s) d s}\right|_{s=t_{0}}+\int_{t_{0}}^{t_{1}} \widehat{\Pi} e^{\int f_{1}(s) d s} d s\right] \\
& S\left(t_{2}^{-}\right)=\frac{1}{\left.e^{\int f_{1}(s) d s}\right|_{s=t_{2}}}\left[(1-p)\left[\left.(1-p) S\left(t_{0}\right) e^{\int f_{1}(s) d s}\right|_{s=t_{0}}+\int_{t_{0}}^{t_{1}} \widehat{\Pi} e^{\int f_{1}(s) d s}\right]+\int_{t_{1}}^{t_{2}} \widehat{\Pi} e^{\int f_{1}(s) d s} d s\right] \\
& S\left(t_{k}^{-}\right)=\frac{1}{\left.e^{\int f_{1}(s) d s}\right|_{s=t_{k}}}\left[\left.(1-p)^{k} S\left(t_{0}\right) e^{\int f_{1}(s) d s}\right|_{s=t_{0}}+\sum_{j=0}^{k-1}(1-p)^{j} \int_{t_{k-j-1}}^{t_{k-j}} \widehat{\Pi} e^{\int f_{1}(s) d s} d s\right] .
\end{aligned}
$$

Let $\varepsilon$ be the smallest integer such that, for $t \geq t_{\varepsilon}$, for which all population approximately at their equilibrium values. Then for $t>t_{\varepsilon}, f_{1}(t) \approx c_{1}$ for $k \geq \varepsilon$,

$$
\begin{gathered}
S\left(t_{k}^{-}\right) \approx \frac{\left.(1-p)^{k} S\left(t_{0}\right) e^{\int f_{1}(s) d s}\right|_{s=t_{0}}}{e^{c_{1} t_{k}}}+\frac{\sum_{j=0}^{\varepsilon-1} \widehat{\Pi}(1-p)^{k-\varepsilon+j} \int_{t_{\varepsilon-j-1}}^{t_{\varepsilon-j}} e^{\int f_{1}(s) d s} d s}{e^{c_{1} t_{k}}} \\
+\sum_{j=0}^{k-\varepsilon-1} \frac{\widehat{\Pi}(1-p)^{j}\left(e^{c_{1} t_{k-j}} e^{c_{1} t_{k-j-1}}\right)}{c_{1} e^{c_{1} t_{k}}} \\
=\frac{\left.(1-p)^{k} S\left(t_{0}\right) e^{\int f_{1}(s) d s}\right|_{s=t_{0}}}{e^{c_{1} t_{k}}}+\widehat{\Pi}(1-p)^{k-\varepsilon} \frac{\sum_{j=0}^{\varepsilon-1}(1-p)^{j} \int_{t_{\varepsilon-j-1}}^{t_{\varepsilon-j}} e^{\int f_{1}(s) d s} d s}{e^{c_{1} t_{k}}}
\end{gathered}
$$




$$
\begin{gathered}
+\frac{\sum_{j=0}^{k-\varepsilon-1} \widehat{\Pi}(1-p)^{j} e^{c_{1} \tau}\left(1-e^{-c_{1} \tau}\right)}{c_{1}} \\
=\frac{\left.(1-p)^{k} S\left(t_{0}\right) e^{\int f_{1}(s) d s}\right|_{s=t_{0}}}{e^{c_{1} t_{k}}}+\widehat{\Pi}(1-p)^{k-\varepsilon} \frac{\sum_{j=0}^{\varepsilon-1}(1-p)^{j} \int_{t_{\varepsilon-j-1}}^{t_{\varepsilon-j}} e^{\int f_{1}(s) d s} d s}{e^{c_{1} t_{k}}}
\end{gathered}
$$

$$
+\frac{\widehat{\Pi}\left(1-e^{-c_{1} \tau}\right)}{c_{1}}\left(\frac{1-(1-p)^{k-\varepsilon} e^{-c_{1} \tau(k-\varepsilon)}}{1-(1-p) e^{-c_{1} \tau}}\right)
$$

As $\quad k \rightarrow \infty,(1-p)^{k} \rightarrow 0,(1-p)^{(k-\varepsilon) \rightarrow 0}$ and $e^{-c_{1} \tau(k-\omega)} \rightarrow 0$, so:

$$
\begin{aligned}
S^{*}\left(t_{k}^{-}\right) & \rightarrow \frac{\widehat{\Pi}\left(1-e^{-c_{1} \tau}\right)}{c_{1}}\left(\frac{1}{1-(1-p) e^{-c_{1} \tau}}\right) \\
& =\frac{\widehat{\Pi}}{c_{1}}\left(1-\frac{p e^{-c_{1} \tau}}{1-(1-p) e^{-c_{1} \tau}}\right) .
\end{aligned}
$$

For the Vaccinated population

$$
\frac{d V}{d t}=\theta S-(\mu+\omega+\alpha \nu I) V .
$$

Assuming $f_{2}(t)=\mu+\omega+\alpha v I$,

$$
-\frac{d V}{d t}=\theta S-f_{2}(t) V
$$

By using impulsive effects and setting $t=t_{k+1}$,

$$
\begin{gathered}
\frac{d V}{d t}=\frac{\theta \widehat{\Pi_{1}}}{c_{1}}\left(1-\frac{p e^{-c_{1} \tau}}{1-(1-p) e^{-c_{1} \tau}}\right)-(\mu+\omega+\alpha v I) V \\
\leq \frac{\theta \Pi_{1}}{c_{1}}\left(1-\frac{p e^{-c_{1} \tau}}{1-(1-p) e^{-c_{1} \tau}}\right)-(\mu+\omega) V .
\end{gathered}
$$

As $p \rightarrow 1$ and $\tau \rightarrow 0$ then $\frac{1-e^{-c_{1} \tau}}{1-(1-p) e^{-c_{1} \tau}} \rightarrow 0$, the upper and lower bounds $\frac{d V}{d t}$ will converges to $\frac{\theta \widehat{\Pi_{1}}}{c_{1}}-(\mu+\omega) V(t)$.

$$
\begin{gathered}
\frac{d I}{d t}=\beta S I-\mu I+\alpha v I V \\
=[(\beta S+\alpha v V)-\mu] I \\
\leq\left[\frac{\beta \lambda(\mu+\omega)+\alpha \theta v \lambda-\mu^{2}(\mu+\omega)}{\mu(\mu+\omega)}\right] I \\
I(t) \leq e^{\widehat{\Pi}_{2} t}
\end{gathered}
$$

where $\left[\frac{\beta \lambda(\mu+\omega)+\alpha \theta v \lambda-\mu^{2}(\mu+\omega)}{\mu(\mu+\omega)}\right]=\widehat{\Pi}_{2}$.

$\tau$ can be isolate to obtain the other identity and hence

$$
\begin{aligned}
\frac{\theta \lambda}{\mu(\mu+\omega)} & =\frac{\widehat{\Pi}_{1}}{c_{2}}\left(1-\frac{p e^{-c_{2} \tau}}{1-(1+p) e^{-c_{2} \tau}}\right) \\
\Rightarrow \tau & =\frac{1}{c_{2}} \ln \left[\frac{p \widehat{\Pi}_{1} \mu(\mu+\omega)-(1+p) c_{2} \theta \lambda}{\widehat{\Pi}_{1} \mu(\mu+\omega)-c_{2} \theta \lambda}\right]
\end{aligned}
$$

As $p \rightarrow 1$ and $\tau \rightarrow 0, I(t)$ converges to 0 if $\frac{\lambda[\beta(\mu+\omega)+\alpha \theta v]}{\mu^{2}(\mu+\omega)}<1$.

Population count in extreme cases

In this section the effect of perfect adherence of the drug on the susceptible and infected population can be verified. The following lemmas are very much important to find the extreme population count.

Lemma 1: For $x(t)$ satisfying $x^{\prime}(t)<c-q(\phi) x(t)$ where $c$ is a constant and $q(\phi)$ is independent of $\mathrm{x}$ and $\mathrm{t}$, then,

1. $x(0)<\frac{c}{q(\phi)} \Rightarrow x(t)<\frac{c}{q(\phi)}$, for all $t$,

2. If $x(0)<\frac{c}{q(\phi)}$ and $\lim _{\phi \rightarrow 0} q(\phi)=\infty \Rightarrow x(t) \rightarrow 0$ as $\phi \rightarrow 0$ for all $t$.

Proof: See the Lemma 4.1 of [13] for the proof.

Lemma 2: Suppose $x(t)$ is a variable satisfying $x^{\prime}(t)>$ $c(t)-q x(t)$ where $\mathrm{q}$ is a constant and $c(t)$ is bounded as $t \rightarrow \infty$. Hence $x(t)>x(0) e^{-q t}+\frac{c(t)}{q}-\frac{1}{q} \int_{0}^{t} c^{\prime}(u) e^{-q(t-u)} d u \rightarrow$ $\frac{c_{\infty}}{q}$, as $t \rightarrow \infty$, with $c_{\infty}=\lim _{t \rightarrow \infty} c(t)$.

Proof: See the Lemma 2 of [11] for the proof.

Remark: If the above inequality are reversed then lemma also holds. Let $N(t)$ be the total population such that $N=S+I+V$. Then

$$
\begin{gathered}
\frac{d N}{d t}=\frac{d S}{d t}+\frac{d I}{d t}+\frac{d V}{d t}=\lambda-\mu(S+I+V) \\
\Rightarrow S+I+V \leq \frac{\lambda}{\mu}
\end{gathered}
$$

Hence the extreme value of the total population is $\frac{\lambda}{\mu}$.

Now, for the infected population

$$
\begin{gathered}
\frac{d I}{d t}=\beta S I+\alpha v I V-\mu I \\
\leq(\mu+\alpha v) \frac{\lambda}{\mu}-\mu I \\
\Rightarrow I \leq(\mu+\alpha v) \frac{\lambda}{\mu^{2}}+\left[I(0)-(\mu+\alpha v) \frac{\lambda}{\mu^{2}}\right] e^{-\mu t} \\
\Rightarrow I(t) \leq(\mu+\alpha v) \frac{\lambda}{\mu^{2}}
\end{gathered}
$$

Also, for the vaccinated population

$$
\begin{aligned}
\frac{d V}{d t}= & \theta S-\alpha \nu I V-(\mu+\omega) V \\
& \leq \frac{\theta \lambda}{\mu}-(\mu+\omega) V \\
& \Rightarrow V(t) \leq \frac{\theta \lambda}{\mu(\mu+\omega)}
\end{aligned}
$$

Hence the extreme values for infected population and vaccinated population are $(\mu+\alpha \nu) \frac{\lambda}{\mu^{2}}$ and $\frac{\theta \lambda}{\mu(\mu+\omega)}$ respectively. 


\section{Numerical Simulation}

In numerical simulation, the perfect drug adherence of rota virus vacccination have been described. All the parameters are taken from Table 1 . The initial condition are assumed as $S(0)=200, I(0)=80, V(0)=0$ and the unit of the concentration is person/day.

Table 1. List of parameters for system (1), and (6).

\begin{tabular}{lll}
\hline Parameter & Definition & Value (Units) \\
\hline$\lambda$ & Recruitment rate & $5\left(\right.$ Personday $\left.^{-1}\right)$ \\
$\omega$ & Rate at which vaccine waves & $0.15\left(\mathrm{~mm}^{-3}\right.$ day $\left.^{-1}\right)$ \\
$\theta$ & Vaccine uptake rate & $0.3-0.7\left(\right.$ day $\left.^{-1}\right)$ \\
$\mu$ & Natural death rate & $0.02\left(\right.$ day $\left.^{-1}\right)$ \\
$\beta$ & Infection rate & $0.02\left(\right.$ Person day $\left.^{-1}\right)$ \\
$\nu$ & Vaccine efficacy & $0.7($ unitless $)$ \\
$\alpha$ & Infection death rate & $0.1($ day \\
\hline
\end{tabular}
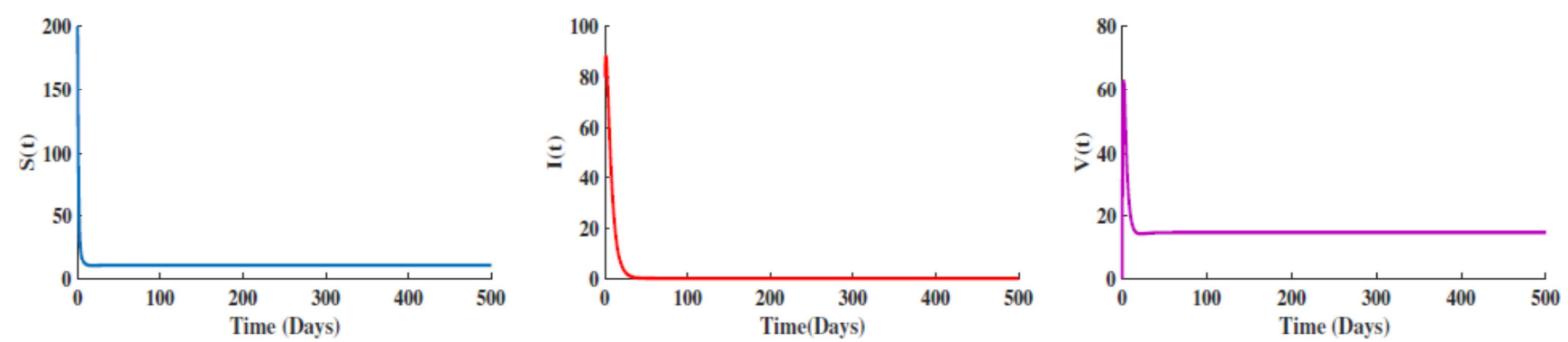

Figure 2. Change of concentration with respect of time in absence of impulsive vaccination. Parameters are valued together with the initial conditions are as in Table 1 with these parametric conditions $R_{0}<1$. Thus the disease free equilibrium exists.
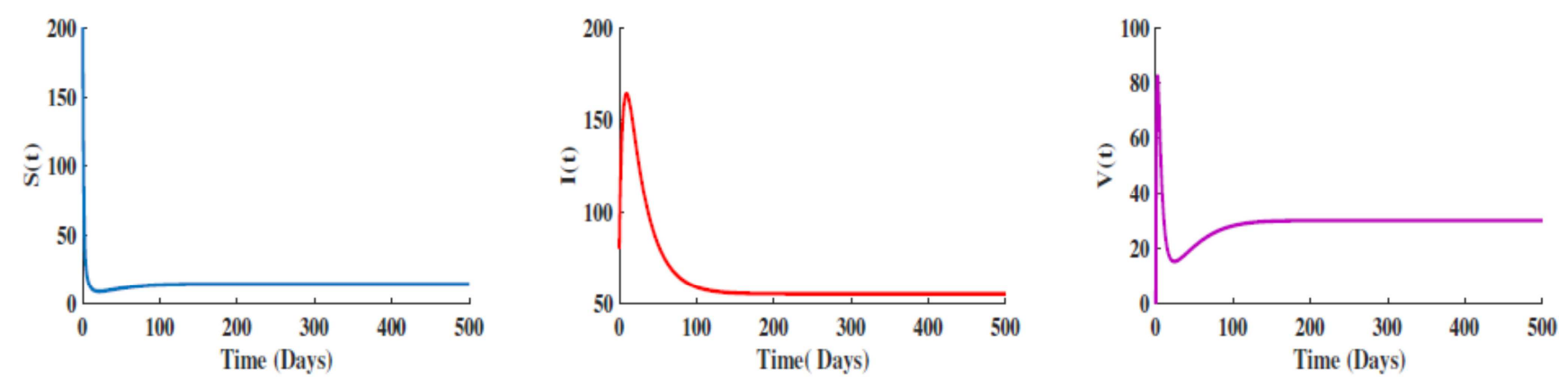

Figure 3. Change of concentration with respect of time in absence of impulsive vaccination. Parameters are valued together with the initial conditions are as in Table 1 with these parametric conditions $R_{0}>1$. Thus the disease free equilibrium exists.

It has been observed that for a high natural death rate $(\mu=0.1), R_{0}<1$, i.e. the disease free state is stable. However, for the low natural death rate $(\mu=0.05), R_{0}>1$ and the system moves towards instability. Thus infected population increases and as a result the system moves from disease free to endemic state. The change of concentration with respect of time in the absence of impulsive vaccination reflects in Figure 3 for the conditions $R_{0}=2.5>1$. Thus, the endemic equilibrium exists. Figure 4 . shows the change

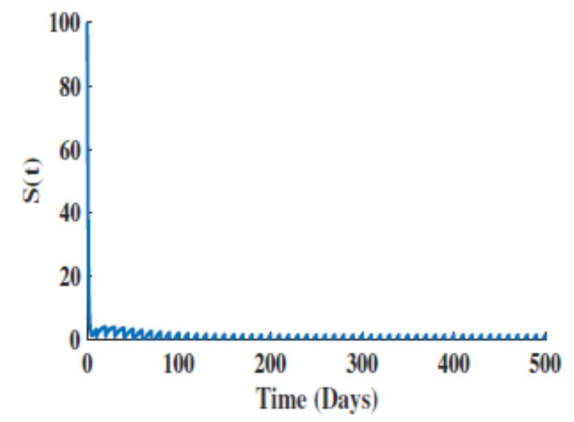

of concentration for each model variables with respect of time in presence of perfect adherence. In this figure the dosing interval is fixed at $\tau=10$. This means the vaccine is taken at ten days interval. From this figure we can easily conclude that after introducing the vaccination, the susceptible population as well as infected population reduces. Also during the treatment the vaccinated population enhance comparatively with the presence of perfect drug adherence.

Figure 4. The impulsive system behavior for the perfect adherence with $\tau=10$. 


\section{Conclusion}

A basic mathematical model has been formulated to study the infection for Rota Virus on human population. In this model, susceptible population, infected population and vaccinated population have been considered. It has been observed that the system has two equilibriums, one is disease free and another is endemic equilibrium. The disease free equilibrium is asymptotically stable if the basic reproduction number is below the unity. But if the basic reproduction number is more than unity, then the disease free equilibrium becomes unstable.

The impulsive differential equations have also been formulated as the vaccination period is not instantaneous. It has also been worked on the optimal interval of vaccination period and weightage of the dosage, by using the impulsive differential equation so that disease can be controlled.

In a nutshell, vaccination against Rota Virus can reduce the infectability of the disease, but is unable to eradicate the disease. The critical vaccination period suggests that careful follow-up must be taken. Hence optimal level of vaccination period as well as weightage of pulse vaccination affect the disease progression and disease replication.

\section{References}

[1] Tate JE, Burton AH, Boschi-Pinto C, Steele AD, Duque J, Parashar UD. "Estimate of worldwide rotavirus-associated mortality in children younger than 5 years before the introduction of universal rotavirus vaccination programmes: a systematic review and meta-analysis," Lancet Infect Dis, V 11, pp. 1-6, (2011).

[2] Jit M, Edmunds WJ., "Evaluating rotavirus vaccination in England and Wales. Part II. The potential cost-effectiveness of vaccination." Vaccine, V25 25, pp. 3971-3979, (2007).

[3] Jaewoo P., Joshua G., Murali H., Matthew F., “An ensemble approach to predicting the impact of vaccination on rotavirus disease in Niger", Vaccine, V35 (43), pp. 5835-5841, (2017).
[4] Onyango L. O., Chuncheng W., Xiaoping X., Owuor G. L., "Modeling the effects of vaccination on rotavirus infection", Advances in Difference Equations, pp. 01-12, (2015)

[5] Parashar, U. D., Bresee, J. S., Gentsch, J. R., Glass, R. I., "Rotavirus", Emerging infectious diseases, V 4(4), pp. 561570, (1998).

[6] Shim, E., Banks, H. T., C. Castillo-Chaves, C., Chavez, A., "Seasonality of Rotavirus Infection with its Vaccination"., Contemp.Math, 410, 327-347, (2006).

[7] Katherine E. Atkinsa, Eunha S., Virginia E. Pitzer Alison P. Galvani, "Impact of rotavirus vaccination on epidemiological dynamics in England and Wales," Vaccine, V 30, pp. 552-564, (2012).

[8] Shim, E., Feng, Z., Martcheva, M., Castillo-Chavez, C., “An age-structured epidemic model of rotavirus with vaccination." Journal of Mathematical Biology, V 53, pp. 719-746, (2006).

[9] Christopher, M., Kribs, Z., Â·Jean-FranÃßois J., Philippe V., Sandrine C., "Modeling Nosocomial Transmission of Rotavirus in PediatricWards," Bull Math Biol, V 73, pp. 1413-1442, (2011).

[10] Roldaoa, A, Helena L. A., Vieiraa, Manuel J. T. Carrondoa, b, Paula M. Alvesa, R. Oliveira, "Rotavirus-Like Particle Production: Simulation of Protein Production and Particle Assembly" 16th European Symposium on Computer Aided Process Engineering and 9th International Symposium on Process Systems Engineering, pp. 1673-1678.

[11] R. J. Smith? Explicitly accounting for antiretroviral drug uptake in theoretical HIV models predicts long-term failure of protease-only therapy, Journal of Theoretical Biology, V 251(2), pp. 227-237, (2008).

[12] R. J. Smith and L. M. Wahl, Drug resistance in an immunological model of HIV-1 infection with impulsive drug effects, The Bulletin of Mathematical Biology, V 67:4, pp. 783-813, (2005).

[13] R. J. Smith and L. M. Wahl, Distinct effects of protease and reverse transcriptase inhibition in an immunological model of HIV-1 infection with impulsive drug effects, The Bulletin of Mathematical Biology, V 66:5, pp. 1259-1283, (2004). 